\title{
Perspective duration of a plane reversible figure
}

JOHN R. PRICE

THE UNIVERSITY OF WESTERN AUSTRALIA

The reversal rate of a plane reversible figure is examined by measuring the duration of each phase separately. The mean duration of the first percept (PI) is found to decline over occasions while that of the reversal phase $(P 2)$ remains constant. It is concluded that the process underlying reversals can not apply equally to both phases.

The results of reversible figure experiments are usually reported in terms of the number of reversals observed over trials. However, the duration of trials varies widely from one study to another and accordingly, direct comparison of results is often difficult to achleve. An alternative method is to take a continuous record of the duration of each occasion of each perspective for a given number of reversals, and plot the mean duration over occasions. In this way a more detailed examination of the reversal rate is posstble, and the problem of trial duration does not arise.

The purpose of the present experiment was to study reveraal rate by the proposed method, and also to investigate an incidental finding by Howard (1961) which sugzests an inverse relationship between stereoscopic vision and the latency of the first reversal.

\section{Apparams}

A copy of the Beaunis "pile of cubes" figure was reproduced from Warren (1919) for the experiment. This tigure was chosen to reduce the possible effects of familiarity and past experience. The stimulus was drawn in black India ink on a plain white card to overall dimensions of 3 in. $x 3$ in., and mounted in a vlewing tunnel $40 \mathrm{in}$. long and $6 \mathrm{in}$. square. Ss viewed the figure binocularly through a 3 in. $x 1$ in. aperture. Ilumination was provided by a concealed Philips $8 \mathrm{~V}$, $50 \mathrm{~W}$ projector lamp set in the viewing end of the tunnel.

Responses were recorded on a moving paper tape. Pressure on a Morse key operated an electromagnet which offset a recording pen until the key was released. A Palmer 50 cycle, $240 \mathrm{~V}$ recorder was set at a constant paper speed of $1 / 2$ in. per sec for recording purposes. The lighting and recorder were operated from the same switch.

\section{Subjects and Procedure}

The Ss were 33 students from the introductory psychology course at the University of Western Australia. The experiment was conducted as part of the formal course requirements and the Ss were drawn from a single laboratory class. Testing was conducted over a period of two weeks, at times arranged to suit the convenience of both $\mathrm{S}$ and $\mathrm{E}$. Before the experiment, each $S$ was tested for stereoscoplc vision on the Bausch and Lomb Orthorater stereopsis test.

The experiment was conducted in a sound-proof room.

The following instructions were read to S: "I am going to show you a reversible figure in the form of a pile of cubes. The figure can be seen as either six or seven cubes (cut out figures were used to demonstrate the two possibilities). I want you to keep your eyes fixated on the center of the flgure, and press this key when there appear to be seven cubes in the pile. Hold the key down until the Agure reverts to a six-cube pile, and then relesse it. Keep doing this until I tell you to stop. Remember to hold the key down as long as you see seven cubes, and release it when there appear to be six."

Each S was tested individually for the time taken to observe 11 reversals, 1.e., 11 occasions of the seven-cube figure, under continuous binocular viewing conditions.

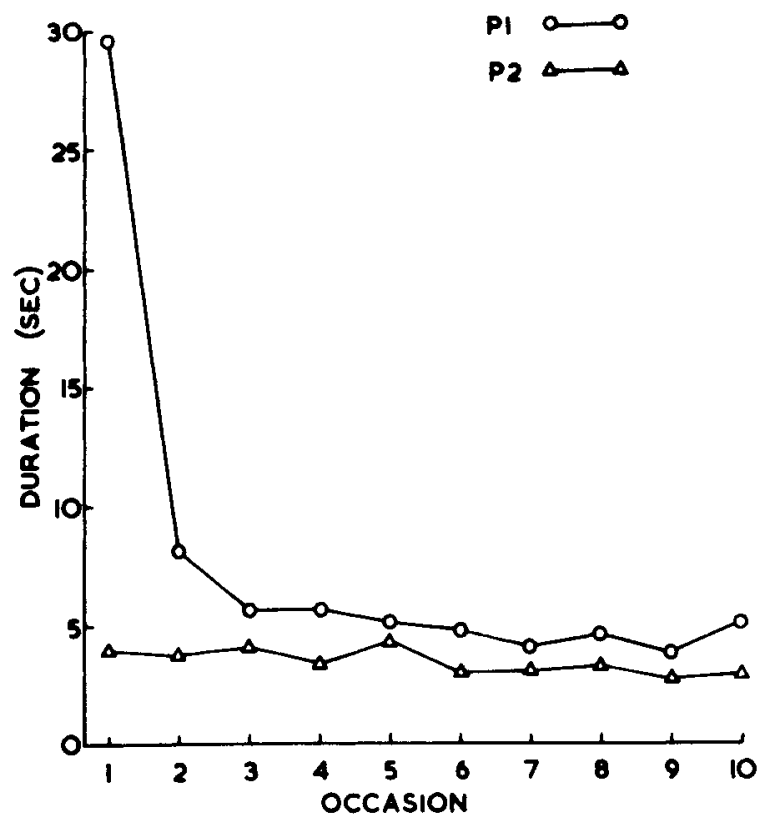

Fig. 1. Mean duration of the initial percept (PI) and reversal phase (P2) over 10 occasions. $N=33$. 


\section{Results and Discussion}

The mean duration of the six-cube percept (P1) and the seven-cube percept (P2) on each occasion is plotted in Fig. 1. Essentially the same curves were obtained by plotting median durations rather than means, except that on the first occasion of P1 the median time was somewhat shorter. The mean latency of the first reversal was $29.6 \mathrm{sec}$, compared with the median value of $20.1 \mathrm{sec}$.

The correlation between latency of the first reversal and Orthorater stereopsis scores was $\mathbf{- 0 . 0 2}$. This is considerably lower than would be expected on the basis of Howard's report that two Ss without stereoscopic vision recorded very brief latencies with the skeletal cube. The fact that his Ss were tested on the Howard-Dolman apparatus suggests that the difference in findings may reflect differences in the two measures of stereoscopic vision.

It may be seen from Fig. 1 that the reversal rate for this stimulus consists of two components which would be confounded by recording only the number of reversals. Similar curves have been found with a rotating skeletal cube (Price, in press), and with the Schroeder staircase. In each case the reversal phase (P2) remained constant in duration, while that of P1 decreased curvilinearly over occasions.

The above results have important implications for research in this area of perception. The reversal rate for most reversible figures tends to increase at a negatively accelerated rate with observation time, and Fig. 1 indicates that this increase is due entirely to the decline of $\mathrm{P} 1$. This has particular relevance to those theories which assume that the process underlying reversals applies equally to both phases, e.g., those of Brown (1962) and Orbach, Ehrlich, \& Heath (1963).

If the initial latency represents the time taken for the process to develop to a level sufficient to induce the first reversal from $P 1$ to $P 2$, the same process cannot account for the change from $P 2$ to $P 1$. In order to do so it would be necessary either for the threshold levels for the two phases to be vastly different, or for the rate of development of the process to be much more rapid during the reversal phase.

In addition, it is difficult to explain the constant duration of $\mathrm{P2}$ in these terms. If the same process were involved in both changes, similar curves would be expected. To produce an increasing reversal curve the duration of at least one phase must decrease and if the same factor determined both aspects of reversal, the decline in duration should also apply to both phases. Such is clearly not the case, and it may be concluded that the above results cannot be explained in terms of a single factor such as the "satiation" or "adaptation" of each of the alternative percepts.

\section{References}

BROWN, K. T. Complete interocular transfer of an adaptation process responsible for perceptual fluctuations with an ambiguous visual figure. Vis. Res., 1962, 2, 469-475.

HOWARD, I. P. An investigation of a satiation process in reversible perspective of revolving skeletal shapes. Quart. J. exp. PsychoL, 1961, 13, 19-33.

ORBACH, J., EHRLICH, D., \& HEATH, A. Reversibility of the Necker Cube: I. An examination of the concept of "satiation of orientation". Percept. mot. Skills, 1963, 17, 439-458.

PRICE, J. R. Two components of reversal rate for a rotating skeletal cube: "conditioned satiation". Aust. J. Psychol, in press.

WARREN, H. C. Human psychology. London: Constable, 1919. 